\title{
Energy nanomaterials: bridging the gap between fundamental science and applied aspects
}

\author{
Nitin Chopra \\ Editor-in-Chief, Nanomaterials and Energy; Associate Professor, \\ Metallurgical and Materials Engineering, Adjunct Professor, Department of \\ Biological Sciences, Affiliated faculty, Center for Materials for Information \\ Technology (MINT), Box 870202, The University of Alabama, Tuscaloosa, \\ AL 35401, USA
}

Understanding the fundamental mechanisms of harvesting energy using nanoscale materials is a primary challenge in the development of alternative energy technology. For example, understanding the relationship between processing and structure of thermoelectric materials could allow higher figure of merits and lead to better materials and devices with improved performance. Similarly, for electrocatalytic splitting of water, it is important to study novel nanoscale electrodes for their basic electrochemical and catalytic properties while, for battery applications, it is critical to develop lithium $(\mathrm{Li})$ extraction processes from natural resources and synthetic media for absorbing Li. These are only a few examples of challenges and demands in the emerging field of energy nanomaterials.

In this issue of Nanomaterials and Energy, we present research in the area of thermoelectric materials, electrochemical nanocatalysts and Li-absorbing ceramic media. The review article by Jariwala and Ravindra ${ }^{1}$ focuses on the processing, properties, and performance evaluation to enhance the figure of merit of chalcogenide-based thermoelectric materials. This article also focuses on the various factors influencing thermoelectric figure of merit. Material systems including bismuth telluride, antimony telluride and lead telluride are discussed and meticulously evaluated for their performance. A research article by Fuqiang et al. ${ }^{2}$ demonstrates the fabrication and output performance of a bismuth telluride-based thermoelectric module. The fabrication approach for this module is based on zone melting method. Figure of merits of 1.1 and 1.09 are achieved at different device-operating temperatures. An output power of $\sim 8.2 \mathrm{~W}$ is achieved in the fabricated device. The power output is reported as a function of temperature difference in the device. Focusing on the importance of structure-function relationships with these two articles, this issue additionally presents articles on electrochemical nanocatalysts for oxygen evolution reaction by $\mathrm{Li}$ et al. ${ }^{3}$ This article demonstrates the growth of $\mathrm{CuO}$ nanowires decorated with $\mathrm{Co}_{3} \mathrm{O}_{4}$ nanoparticles, material characterisation, as well as examination of their electrochemical behaviour. The heterostructures demonstrate improved catalytic response for oxygen evolution reaction. Finally, the article by Hajipour et al. ${ }^{4}$ shows the material development for storage and extraction of Li from brine. In this regard, the synthesis of nanostructured zirconium oxide is reported, and $\mathrm{Li}$ ion absorption studies as well as various structural and analytical characterisation studies are performed. The uptake of lithium ions into the developed media is observed to be $17 \mathrm{mg} / \mathrm{g}$.

Overall, this issue of Nanomaterials and Energy presents a set of unique studies, which will critically advance our knowledge in the specific areas of thermoelectrics, electrocatalysis, and $\mathrm{Li}$ ion extraction and storage. With this issue, I also encourage readers and interested scientific community to submit their novel research work in the areas of energy nanomaterials to this journal. One of the aims of Nanomaterials and Energy is to provide an exciting platform for publishing high-quality energy research that bridges the gap between fundamental science and applied aspects.

\section{REFERENCES}

1. Jariwala, B.; Ravindra, N. M. Process, property and performance of chalcogenide-based thermoelectric materials. Nanomaterials and Energy 2014, 3 (3).

2. Fuqiang, C.; Yanji, H.; Chao, Z. Fabrication and output power test of a $\mathrm{Bi}_{2} \mathrm{Te}_{3}$-based thermoelectric module. Nanomaterials and Energy 2014, 3 (3).

3. Li, Y.; Kumar, K.; Chopra, N. Fabrication and electrochemical properties of copper oxide $(\mathrm{CuO})$ nanowire-cobalt oxide $\left(\mathrm{Co}_{3} \mathrm{O}_{4}\right)$ nanoparticle heterostructures for oxygen evolution reaction. Nanomaterials and Energy 2014, 3 (3).

4. Hajipour, H.; Moazeni, M.; Askari, M.; Nusheh, M. Adsorption behavior of $\mathrm{Li}^{+}$from aqueous medium using zirconium oxide nano-ion sieve. Nanomaterials and Energy 2014, 3 (3). 